\title{
A Response to 'Investigating Emotional Similarity: A Comment on Riberto, Pobric and Talmi (2019)'
}

\author{
Martina Riberto ${ }^{1,2} \cdot$ Gorana Pobric $^{1} \cdot$ Deborah Talmi $^{3}$
}

Received: 31 March 2020 / Accepted: 31 March 2020 / Published online: 15 April 2020

○) Springer Science+Business Media, LLC, part of Springer Nature 2020

We thank Levine and Wackerle (2020) for their response to our literature review, 'The emotional facet of subjective and neural indices of similarity'. They raised an excellent point- that it is important to take into account the control of possible confounding factors in light of the specific research question. For example, in studies primarily interested in individual differences in the representational space, it is not necessary to control for visual and semantic similarity during the stimulus selection; such control could even be detrimental if it severely decreases the sample.

We agree with the authors that it is challenging to exert experimental control over complex scenes, and that therefore, the eventual level of experimental control is often a compromise between the desirable and the feasible. For example, while available datasets are easier to use, they also present significant limitations. To overcome them, in a recent unpublished study we selected 72 complex pictures that depicted 4 different outdoor situations (4 categories, 18 pictures per category) by using different sources [e.g. Emopics (Wessa et al. 2010), NAPS (Marchewka et al. 2014), Google images]. According to the results from Levine and Wackerle (2020) reported in Fig. 1(B), 18 pictures per category should be enough to sample the representational space

Deborah Talmi and Gorana Pobric are joint senior authors.

Martina Riberto

martina.riberto@manchester.ac.uk

Gorana Pobric

gorana.pobric@manchester.ac.uk

Deborah Talmi

dt492@cam.ac.uk

1 Division of Neuroscience and Experimental Psychology, University of Manchester, Oxford Rd, Manchester M13 9PL, UK

2 Department of Neurobiology, Weizmann Institute of Science, Herzl St 234, Rehovot, Israel

3 Department of Psychology, University of Cambridge, Downing St, Cambridge CB2 3EB, UK and avoid spurious correlations. Yet creating this stimulus sample required substantial effort, which will not be justified in every case.

We also agree that the methodological concerns that we discussed depend on the goal of the study. When researchers are interested in individual differences between participants in the representational space, the factors that we mentioned are not confounding. Yet when authors are interested in individual or group differences in emotional similarity judgements, then it may be beneficial to control for factors that affect the thematic similarity within emotional and neutral stimulus categories. While individuals may feel differently about specific stimuli, it is possible to control for differential thematic similarity (often higher within emotional vs neutral categories) in advance, because such differences depend on shared semantic knowledge. Removing average differences (here, between stimulus categories) can facilitate the interpretation of individual differences.

In conclusion, we appreciated the comments and we thank the authors for the excellent points that they raised. We hope that this exchange will guide researchers during the difficult process of stimulus selection.

\section{References}

Marchewka A, Żurawski Ł, Jednoróg K, Grabowska A (2014) The Nencki Affective Picture System (NAPS): introduction to a novel, standardized, wide-range, high-quality, realistic picture database. Behav Res Methods 46(2):596-610

Wessa M, Kanske P, Neumeister P, Bode K, Heissler J, Schönfelder S (2010) EmoPics: Subjektive und psychophysiologische Evaluation neuen Bildmaterials für die klinisch-bio-psychologische Forschung. Zeitschrift für Klinische Psychologie und Psychotherapie 39(Suppl 1/11):77

Publisher's Note Springer Nature remains neutral with regard to jurisdictional claims in published maps and institutional affiliations. 